de telle sorte que les deux tyrosines du site autophosphorylable (voir schéma médecine/sciences $n_{3}$, vol. I, p. 162 el figure I) sont remplacées par des phénylalanines, le récepteur codé par ce "gène muté " n'est plus "autophosphorylable", son activité de protéine kinase spćcifique des résidus tyrosines de substrats exogènes est bassc... et surtout le transport transmembranaire de glucose stimulć par l'insuline est extrêmement diminuć. Un même résultat est obtenu lorsqu'une délćtion correspondant aux I I2 acides aminćs carboxyterminaux est introduite dans l'ADNc.

Ces résultats prouvent de façon remarquable que l'activité protéine kinasique de la chaine $\beta$ du réceptcur est réguléc par l'autophosphorylation de deux résidus tyrosines et est indispensable à la régulation par l'insuline du transport transmembranaire du glucose. Ia cascade d'événements intervenant dans ce phénomène est résuméc dans la figure $I$ : la fixation de l'insuline sur le récepteur entraîne une autophosphorylation des tyrosines I I62 et I 63 de la chaine $\beta$, qui est cllemême associćc à une stimulation de l'activité protéine kinasique du récepteur sur d'autres substrats. $L_{a}$ phosphorylation de protéines membranaires encore inconnues conduirait à l'activation du transport du glucose. Ce modèle est parfaitement cohérent avec une constatation clinique rapportce il y a deux ans $[2,3]$ : l'autophosphorylation de la chaîne $\beta$ du récepteur est anormalement faible chez certains malades ayant un diabc̀te insulino résistant.

A.K.

I. Elllis L, Clauser F, Morgan OI), Edery M, Roth R, Rutter VJ. Replacement of insulin receptor tyrosine residues i 162 and 1163 compromises insulin-stimulated kinase activity and uptake of 2-deoxyglucosc. Cicll 1986; $+5: 721-32$.

2. Grunberger G, Zick I, Gorden P. Defect in phosphorylation of insulin receptors in cells from an insulin-resistant patient with normal insulin binding. Science 1984; $223: 9.32-4$.

3. Grigorescu F, Flier JS, Kahn RC. I)clect in insulin receptor phosphorylation in erythrocites and fibroblasts associated with screre insulin resistance. 7 Binl Chem 1984; 259 : 15003-6.

Flash! Le gène de la myopathie de Duchenne est immense (plus de I 000 kilobases) et code pour un très grand messager de 16 kilobases présent, notamment, dans le muscle fatal (Source: laboratoire de Louis Kunkel, Boston).

\title{
Mécanismes post-traductionnels d'activation des oncogènes
}

Une activité 'biologique peut être régulée à de très nombreux niveaux; les mécanismes de régulation de la transcription des gènes, de la maturation et de la stabilité des ARN messagers ont été analysés en détail dans les numéros précédents de médecine/sciences. Nous avons notamment signalé la jréquence avec laquelle la stabilisation de messagers codant pour des oncogènes pouvait être responsable de leur activation (médecine/sciences $n^{\prime} 5$, vol. 2, p. 286). La traduction de l'ARNm en protéine peut également être contrôlée, comme l'indique éloquemment l'exemple de I ARN du virus $L A V$ récemment rapporté (médecine/sciences $\mathrm{n}^{5}$, vol. 2, p. 285). L'activité biologique et la stabilité d'une protéine peuvent-être, enfin, modifiées après sa synthèse par de nombreux phénomènes parmi lesquels, sans être exhaustif, on peut citer la phosphorylation, la glycosylation et lassociation réversible à une autre molécule, ligand de petite taille ou macromolécule. De tels processus post-traductionnels sont en cause dans l'activation de certains oncogènes. Le virus du. polyome (virus à $A D N$ ) comporte dans son génome deux oncogènes dénommés grand $T$ et moyen $T$; le premier appartient plutôt à la calégorie des oncogènes "immortalisants", le second étant "transformant" $[I]$. Le mécanisme de l'action de moyen $T$ est sa liaison avec l'oncogène cellulaire c-src [2] dont il stimule l'activité tyrosine kinasique $[2,3]$. Des gènes mutés moyen $T$ doni les pro- duits ont perdu cette propriété de se complexer à c-src ont également perdu leur pouvoir transformant [4]. $\mathrm{c}-\mathrm{src}$ code pour la protéine $p 60^{\mathrm{c}-\mathrm{src}}$, une protéine kinase spécifique des résidus tyrosines qui, in vivo, est elle-même phosphorylée sur la tyrosine 527, proche de l'extrémité carboxyterminale de la molécule. Par contre, p6ov-src, produit du gène transformant du virus de Rous, est phosphorylée in vivo sur la tyrosine 4i6. Dans les cellules transformées par le virus du polyome. p6o $0^{\mathrm{c}-\text { src }}$ associée au produit de moyen $T$ est, comme la protéine virale p6ov-src, phosphorylée sur la tyrosine $416[5]$. Ces résultats suggèrent par conséquent que la liaison entre p6oc-src et la protéine moyen $T$ entraine la phosphorylation de $p 6 o^{c-s r c}$ sur une autre tyrosine que celle qui est phosphorylée dans les cellules normales, cette modification du site de phosphorylation expliquant l'augmentation de l'activité tyrosine kinasique et la transformation cellulaire.

Un autre virus à $A D N, S V-40$ (simian virus-40), possède dans son génome un seul gène transformant, également appelé grand $T$; son produit est capable de former des complexes spécifiques avec la protéine p53 codée par un oncogène cellulaire (médecine/sciences $\mathrm{n}^{\circ}$ 5, vol. 2, p. 286) et de la stabiliser. Il est fort possible que ce phénomène de stabilisation de p53, sinon très instable, soit à l'origine du pouvoir transformant de l'oncogène $T$ de $S V$-40 [6]. p53

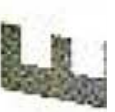


est régulée au cours du cycle cellulaire à au moins deux niveaux, la stabilité de son messager et sa stabilité propre; cette dernière est augmentée par sa liaison avec une protéine cellulaire de 70000 de poids moléculaire dont la concentration varie elle aussi en fonction de l'activité mitotique de la cellule et s'accroît fortement en cas d'augmentation anormale de la température ("protéine de choc thermique ").

Une interaction spécifique entre une autre protéine de choc thermique (la " $90 K$ ", de 90000 de poids moléculaire) et les produits de nombreux oncogènes viraux [v-sarc [7], v-fes, v-fgr (M.G. Catelli, communication personnelle), v-fos, v-yes, v-abl a également été décrite et il est possible que le même type de phénomène intervienne au niveau des produits des oncogènes cellulaires équivalents.

Ainsi, ces mécanismes d'activation des produits pré-existants d'oncogènes cellulaires par des protéines virales ou cellulaires jouent-ils probablement un rôle encore imparfaitement exploré dans la transformation maligne et la régulation de la division cellulaire.

A.K.

I. Mougneau E, Glaichenhaus N, Cuzin F. Analyse génétique des étapes précoces de la progression tumoralc. medecine/sciences 1985; 1 :86-90.

2. Stchelin D. Les oncogènes cellulaires, clés de la cancérogénèsc. médecine/sciences $1985 ; 1: 10-6$.

3. Bolen JB, Thicle CJ, Isracl MA, et al. Enhancement of cellular src gene product-associated tyrosyl kinasc activity following polyoma virus infection and transformation. Cell 1984; 38 : 767-77.

4. Cheng SH, Markland W, Markham AF, Smith AE. Mutations around the NG 59 lesion indicatc an active association of polyoma virus middle $\mathrm{T}$ antigen with $\mathrm{pp}^{\mathrm{sic}} \mathrm{o}^{\mathrm{src}}$ is required for cell transformation. Embo 7 1986; $5: 325-34$

Cartwright CA Kaplan PL, Cooper JA, Hunter T, Eckhart W. Altered sitcs of tyrosinc phosphorylation in pp6o $0^{c-s r c}$ associated with polyoma virus middle tumor antigen. Mol Cell Biol 1986; $6: 1562-70$.

6. Pinhasi-Kimhi O, Michalovitz D, Ben-Zecv Z Oren M. Specific interaction between the $\mathrm{p} 53 \mathrm{cel}-$ lular tumour antigen and major heat shock protcins. Nature 1986; $320: 182-4$

7. Brugge JS. The specific interaction of the

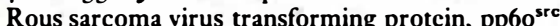
with two cellular protcins. Cell $198 \mathrm{I} ; 25: 363-72$.

\section{Inhibine, FRP et activine}

\author{
Des hormones ovariennes \\ régulant la sécrétion de FSH
}

L'inhibine est une protéine dimérique, secrétée par l'ovaire, qui inhibe la sécrétion hypophysaire de la FSH (Follicle-Stimulating Hormone) sans modifier celle de LH (Luteinizing Hormone). Elle est composée de deux sous-unités, l'une de 18 ooo de poids moléculaire (sous-unité $\alpha$ ), l'autre de 14000 (sous-unités $\beta$ ), liées par un pont disulfure. Il existe

deux types de sous-unité $\beta, \beta_{A}$ et $\beta_{B}$. La séquence protéique de cette hormone est maintenant connue grâce au clonage moléculaire des ADN complémentaires codant pour les différentes sous-unités. Il existe de grandes homologies de structure entre les chaînes $\beta$ de l'inhibine et le TGF- $\beta$ (Transforming Growth Factor, voir nouvelle page 467).

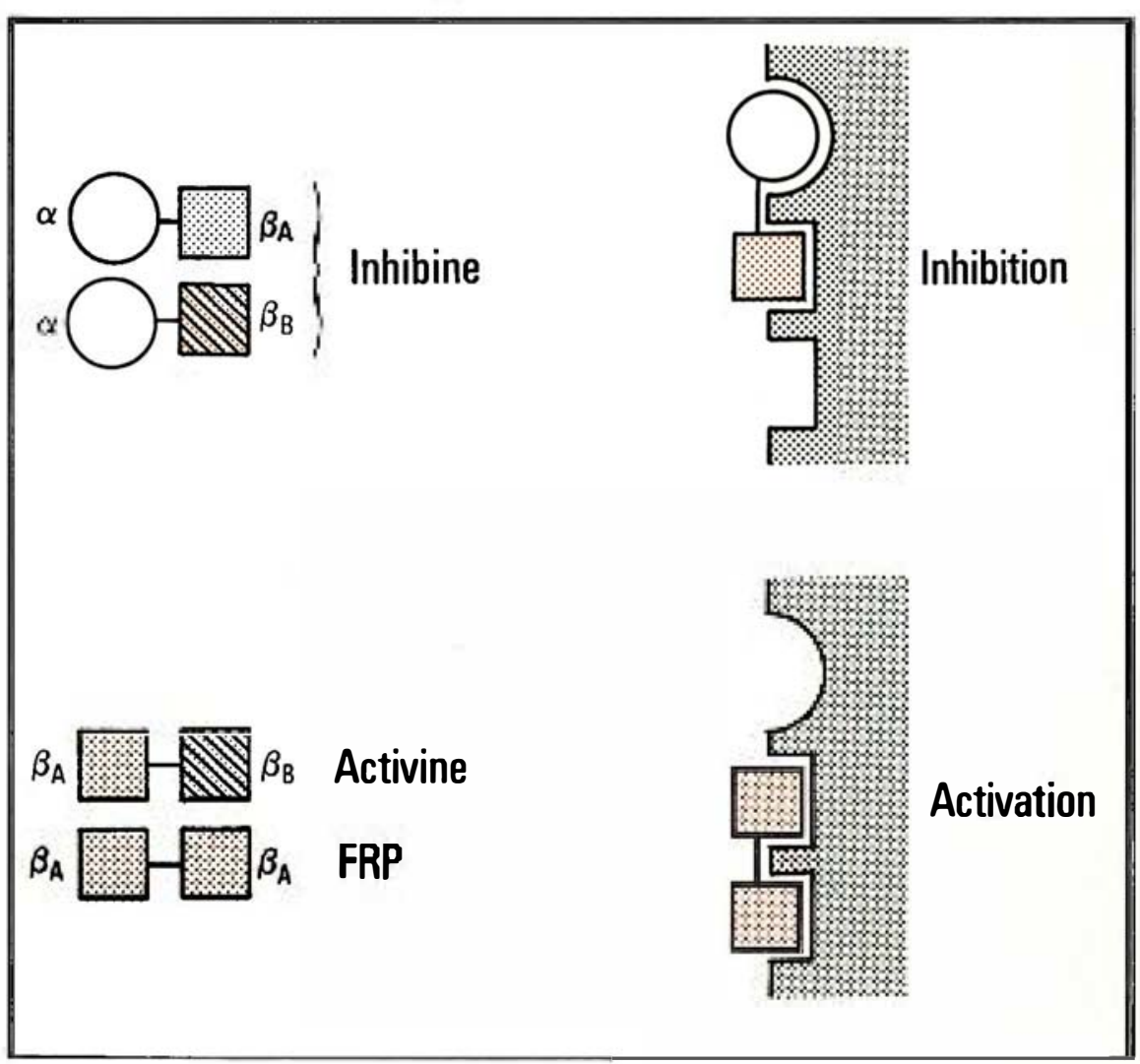

Figure 1. Structure et mode présumé d'action des hormones Inhibine. Activine et FRP. Cette figure fait l'hypothèse que deux types de récepteurs existeraient au niveau de la membrane des cellules cibles, reconnaissant les sous-unités $\alpha$ et $\beta$. La fixation des dimères $\alpha$. $\beta$ entraînerait une inhibition de la secrétion de $F S H$, alors que celle des dimères $\beta$. $\beta$ serait stimulatrice. 